\section{Epstein-Barr virus-negative aggressive natural killer-cell leukaemia with high P-glycoprotein activity and phosphorylated extracellular signal-regulated protein kinases 1 and 2}

\author{
Sanja Perkovic, ${ }^{1}$ Sandra Basic-Kinda, ${ }^{2}$ \\ Vladimir Gasparovic, ${ }^{3}$ Zeljko Krznaric, ${ }^{4}$ \\ Jaksa Babel, ${ }^{3}$ Ivana Ilic, ${ }^{5}$ Igor Aurer, ${ }^{2}$ \\ Drago Batinic ${ }^{1}$
}

1Department of Laboratory Diagnostics, Division of Immunology and Department of Internal Medicine;

${ }^{2}$ Division of Haematology; ${ }^{3}$ Division of Intensive Care Medicine; ${ }^{4}$ Division of Gastroenterology; ${ }^{5}$ Department of Pathology and Cytology, University Hospital Centre Zagreb and School of Medicine, University of Zagreb, Croatia

\section{Abstract}

Aggressive natural killer-cell leukaemia (ANKL) is a rare type of disease with fulminant course and poor outcome. The disease is more prevalent among Asians than in other ethnic groups and shows strong association with Epstein-Barr virus (EBV) and P-glycoprotein (P-gp) expression associated with multidrug resistance. Here we present a case of a 47 year old Caucasian female with a prior medical history of azathioprine treated ulcerative colitis who developed EBV-negative form of ANKL. The patient presented with hepatosplenomegaly, fever and nausea with peripheral blood and bone marrow infiltration with up to $70 \%$ of atypical lymphoid cells positive for cCD3, CD2, CD7, CD56, CD38, CD45, TIA1 and granzyme $\mathrm{B}$, and negative for sCD3, CD4, CD5, CD8, CD34 and CD123 indicative of ANKL. Neoplastic CD56 ${ }^{+}$NK-cells showed high level of P-glycoprotein expression and activity, but also strong expression of phosphorylated extracellular signal-regulated protein kinases 1 and 2 (ERK1/2) MAP kinase. The patient was treated with an intensive polychemotherapy regimen designed for treatment of acute lymphoblastic leukaemia, but one month after admission developed sepsis, coma and died of cardiorespiratory arrest. We present additional evidence that, except for the immunophenotype, leukaemic NK-cells resemble normal NKcells in terms of P-gp functional capacity and expression of phosphorylated ERK1/2 signalling molecule. In that sense drugs that block P-glycoprotein activity and activated signalling pathways might represent new means for targeted therapy.

\section{Introduction}

Mature natural killer (NK) malignancies, according to the World Health Organization classification, comprise extranodal NK/T-cell lymphoma, nasal type (ENKL) and aggressive NK-cell leukaemia (ANKL).1,2 These are rare types of neoplasms, particularly in Western countries, but with somewhat higher incidence rate in Asian and Central and South American population. Both entities have a highly aggressive clinical course with very poor survival rates. ${ }^{3}$

Fernandez et al. ${ }^{4}$ and Koizumi et al. ${ }^{5}$ were the first to report and give evidence of a new clinical entity named aggressive NK-cell leukaemia. Bone marrow and peripheral blood are common sites of involvement, with equal incidence in males and females. Median age of patients diagnosed with ANKL is between 30 and 40 years. Common symptoms comprise fever, anaemia, hepatosplenomegaly, disseminated intravascular coagulation and lymphadenopathy. ${ }^{6}$ Survival rates are extremely low, measuring in weeks and not exceeding 2 years (median 2 months).

Common immunophenotype of aggressive NK-cell leukaemia is surface CD3-negative (sCD3 $\left.{ }^{-}\right)$, cytoplasmic CD3-positive $\left(\mathrm{cCD}^{+}\right)$, CD2-positive (CD2 $\left.{ }^{+}\right), \mathrm{CD} 7$-positive $\left(\mathrm{CD} 7^{+}\right)$, CD56-positive (CD56+), CD5-negative $\left(\mathrm{CD}^{-}\right)$, CD4-negative (CD4-), CD8-negative (CD8 ${ }^{-}$), with germline configuration of T-cell receptor. ${ }^{6}$ Frequent cytogenetic abnormalities are deletion 6q21-q25 and loss of 17p13. ${ }^{7}$ Also, mature NK neoplasms are highly associated with Epstein-Barr virus (EBV), implying a role of this virus in tumour pathogenesis. ${ }^{8}$

It is noteworthy to mention that NK cells have the highest level of P-glycoprotein (P-gp) (MDR-1) expression and activity of all lymphoid cells. ${ }^{9}$ Accordingly, malignancies arising from mature NK cells frequently express $\mathrm{P}$ gp, ${ }^{10,11}$ a protein involved in multidrug resistance mechanism (MDR). This may be a partial answer to the question of why there is such a high chemotherapy failure rate in NK-cell derived neoplasms.

\section{Case Report}

A 47-year-old Caucasian female was admitted to the University Hospital Centre Zagreb, Croatia, in September 2010 with high fever, splenomegaly and urinary infection. She reported feeling nausea, fatigue and pain in
Correspondence: Drago Batinic, Department of Laboratory Diagnostics, Division of Immunology, University Hospital Centre Zagreb and School of Medicine, University of Zagreb,

Kispaticeva 12, HR-10000 Zagreb, Croatia.

Tel. +38.5123.67238 - Fax: +38.5123 .67395$

E-mail:drago.batinic@zg.t-com.hr

Key words: NK-cell leukaemia, P-glycoprotein, ERK MAP kinase, ulcerative colitis.

Funding: this study was supported in part by grants from Croatian Ministry of Science, Education and Sports, number 214-1081347-0355 (PI Drago Batinic), 108-0000000-0180 (PI Vladimir Gasparovic) and 108-1081872-1908 (PI Igor Aurer)

Acknowledgements: the authors thank Koraljka Gjadrov Kuvezdic, MD, PhD for providing figure of May-Grünwald Giemsa staining.

Contributions: PS, BKS, II and BD contributed to conception and design, analysis and interpretation of data and drafting the article; GV, KZ, BJ and AI critically revised the article.

Conflict of interests: the authors report no potential conflict of interests.

Received for publication: 6 March 2012.

Revision received: 22 May 2012.

Accepted for publication: 12 June 2012.

This work is licensed under a Creative Commons Attribution NonCommercial 3.0 License (CC BYNC 3.0).

(OCopyright S. Perkovic et al., 2012

Licensee PAGEPress, Italy

Hematology Reports 2012; 4:e16

doi:10.4081/hr.2012.e16

the lower left abdominal quadrant. Medical history revealed a prior diagnosis of ulcerative colitis (UC) in 2002 after which she was on continuous treatment with topical and peroral aminosalicylates. She received interferon within a clinical trial in 2002 and 2003. The patient was in remission from 2005 until 2008 when exacerbation occurred and corticosteroid therapy was introduced. Due to progression of the disease in 2009 , the patient was started on azathioprine and continued the treatment for 17 months. In May 2010 UC worsened and sideropaenic anaemia developed. She was admitted to hospital, treated with corticosteroids, antibiotics and erythrocyte transfusions with a good response. In 2005 Hashimoto's thyreoiditis was diagnosed and thyroxine therapy initiated.

Upon admission, the patient's laboratory findings were as follows: white blood cells 5.92 
$\times 10^{9} / \mathrm{L}\left(3.4-9.7 \times 10^{9} / \mathrm{L}\right) ;$ haemoglobin $86 \mathrm{~g} / \mathrm{L}$ (119-157 g/L); haematocrit $0.286 \mathrm{~L} / \mathrm{L}(0.356$ $0.470 \mathrm{~L} / \mathrm{L})$; mean corpuscular volume $68.9 \mathrm{fL}$ (83.0-97.2 fL); red blood cells $4.15 \times 10^{12} / \mathrm{L}$ $\left(3.86-5.08 \times 10^{12} / \mathrm{L}\right)$; platelets $164 \times 10^{9} / \mathrm{L}(158$ $\left.42 \times 10^{9} / \mathrm{L}\right)$; other parameters were within normal range (PT, APTT, fibrinogen) or slightly increased (LDH, CRP, AST, ALT, GGT, ALP). Multislice computerised tomography revealed hepatosplenomegaly, with multiplied and enlarged retroperitoneal lymph nodes. Colonoscopy revealed no signs of active UC. Progressive deterioration of haematological parameters occurred, with an increase in white blood cells $96.65 \times 10^{9} / \mathrm{L}$, development of disseminated intravascular coagulation: PT 0.63 (>0.70), APTT $55.2 \mathrm{~s}(24.0-33.0 \mathrm{~s})$, fibrinogen $0.6 \mathrm{~g} / \mathrm{L}(1.8-4.1 \mathrm{~g} / \mathrm{L})$ and $\mathrm{LDH}$ increase $732 \mathrm{U} / \mathrm{L}(<241 \mathrm{U} / \mathrm{L})$.

Peripheral blood and bone marrow smears revealed up to $70 \%$ of atypical lymphoid cells. The morphology (medium/large granular cells, round nucleus, intermediate condensed chromatin, pronounced nucleolus and basophilic cytoplasm) (Figure 1A) and cytochemical pattern $\left(\mathrm{MPO}^{-}, \mathrm{SUDAN}^{-}, \mathrm{ANAE}^{-}, \mathrm{PAS}^{+/}\right)$of these cells was indicative of lymphoproliferative disease. Bone marrow biopsy revealed reduction of all three haematopoietic cell lineages and diffuse interstitial infiltrate of medium sized to large atypical lymphoid cells. Immunohistochemically, the cells were $\mathrm{CD}_{56}{ }^{+}, \mathrm{TIA}^{+}$ (Figures $1 \mathrm{~B}$ and $\mathrm{C}$ ), granzyme $\mathrm{B}+$, cytoplasmic $\mathrm{CD}^{+}, \mathrm{CD}^{-}$and $\mathrm{CD} 20^{-}$. Flow cytometry of bone marrow aspirate revealed relatively homogenous population of cells (65\%) positive for CD45, cCD3, CD2, CD7, CD38, CD56, and negative for sCD3, CD4, CD5, CD8, CD13, CD117, CD34, HLA-DR, TdT and CD123. Karyotyping showed no chromosome abnormalities $(46, \mathrm{XX})$. Considering all of the above, the patient was diagnosed with aggressive NK-cell leukaemia.

Further analysis concentrated on P-gp and phosphorylated signalling molecules in CD56+ neoplastic cells (Figure 2). P-gp activity was assessed using Rhodamine 123 and verapamil as an inhibitor. This functional test demonstrated high P-gp transport activity (ratio of fluorescence intensity, RFI=16.54) which was in concordance with high membrane P-gp expression $(\mathrm{D}=0.63$, Kolmogorov-Smirnov test) by Mrk16 antibody staining (Kamiya Biomedical Company). Monoclonal antibody staining of cytoplasmic phosphorylated molecules Akt, ERK1/2 and p38 revealed strong phosphorylated ERK1/2 expression only ( $\mathrm{D}=0.62$, Kolmogorov-Smirnov test).

Enzyme-linked immuno-sorbent assay testing showed negative serum EBV VCA IgM and EBV EA IgG, and positive EBV VCA IgG and EBNA IgG, which was indicative of past EBV infection. In situ hybridisation for EBV-encoded early small RNA1 (EBER-1) in bone marrow neoplastic cells was negative, as well as EBER1 staining in neoplastic cells found in patient's gastric mucosa.

Upon diagnosis of aggressive NK-cell leukaemia, the patient was treated with a chemotherapy protocol designed for treatment of acute lymphoblastic leukaemia in younger patients consisting of steroids, vincristine, cyclophosphamide, danorubicine and repeated administration of asparaginase. Initially, the patient responded favourably, leukocyte counts dropped, hepatosplenomegaly disappeared and coagulation parameters normalized. During pancytopaenia, signs of meningeal infiltration with leukaemia developed. The patient was treated with repeated intrathecal administration of methotrexate, cytarabine and steroids without improvement. Coma and sepsis ensued and the patient died one month after being admitted to our hospital.

\section{Discussion}

As mentioned above, aggressive NK-cell leukaemia is uncommon disease, but occurs with higher frequency in Asians and Central/South Americans than in other ethnic groups. The vast majority of such cases are associated with EBV which is found in clonal episomal form implying active aetiologic role in NK-cell-derived malignancy development. ${ }^{12}$ Here we emphasize the clinical and scientific importance of reporting much rarely EBV-negative form of ANKL in Caucasian patient. Namely, 13-15\% of all ANKL cases are EBV-negative, raising certain suspicions in regards of proposed pathogenic mechanisms. ${ }^{13,14}$ Consequently, presumptions of different clinical course and outcomes of patients with EBV-positive and EBV-negative ANKL were made. The published reports on this issue show conflicting results: one group reported no significant prognostic value of EBV-positivity, ${ }^{13}$ whereas the other presented evidence of significantly longer survival of EBV-negative ANKL patients in comparison to EBV-positive ones (11.5 vs 1.5 months, respectively). ${ }^{14}$ Obviously, larger cohorts of patients will be more conclusive, since both reports include only two EBV-negative ANKL cases. The patient we are reporting had a very aggressive and rapid clinical course despite EBV-negativity.

Taking into account the poor outcome of aggressive NK-cell leukaemia (median survival $<2$ months), it is important to improve knowledge of pathophysiology of this disease and particularly of the biology of leukaemic NK cells. In this respect, it is well known that NK cells in peripheral blood of healthy individuals have high P-gp expression and activity, the highest of all lymphoid cells. ${ }^{11,12}$ Even though it is known that P-gp acts as a protector of haematopoietic stem cells against toxins, its role in mature NK cells still needs to be elucidated. Our case confirms a strong P-gp activity and expression in malignant NK-cells. Since MDR-1 protein is involved in multidrug resistance mechanism, this might at least partially explain why ANKL is so resistant to chemotherapy. ${ }^{15}$ Accordingly, ANKL therapy protocols could be created in a way that they combine P-gp activity modulators and standard chemotherapy. Alternatively, they could avoid chemotherapeutics known to be Pgp substrates.

Furthermore, signalling pathways are very important in tumorigenesis. Utilisation and constitutive activation of certain signalling molecules enable malignant leukaemic cells to suppress normal haematopoiesis and continu-
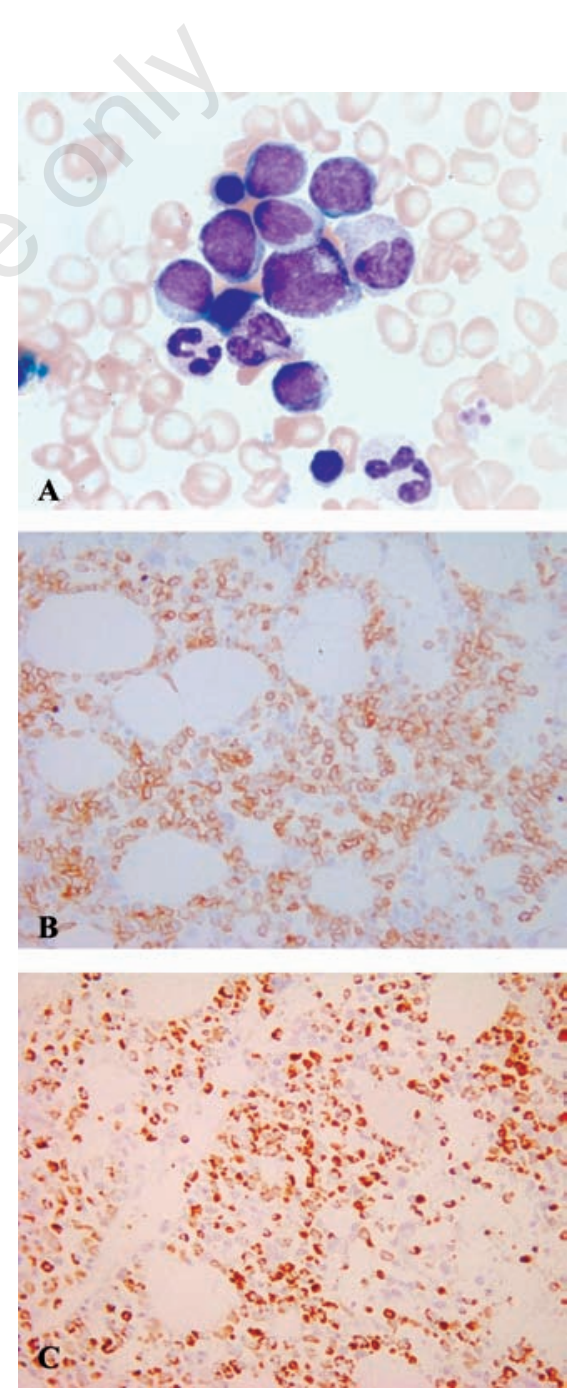

Figure 1. Bone marrow aspirate (A). The blasts have large nuclei with fine chromatin, a single nucleolus, and a moderate amount of light blue cytoplasm (May-Grünwald Giemsa stain, 1000x). Bone marrow biopsy (B, C). Immunohistochemically, tumor cells are $\mathrm{CD}^{\circ} 6^{+}$(B) and show granular cytoplasmic reaction when stained for TIA1 (C) (40x). 
ously proliferate. Peripheral blood NK cells and NK cell lines demonstrate ERK1/2 (p44/p42 mitogen-activated protein kinase) phosphorylation. This pathway plays critical role in NKcell cytotoxicity; it drives lytic activity and mobilisation of granzyme B. ${ }^{16-18}$ However, it has been proposed that there are two different pathways responsible for ERK1/2 activation. Sky/Zap70 - PI3K - Rac - Pak - MEK - ERK cascade regulates human PB NK cell cytolysis, while Ras - Raf - MEK - ERK cascade regulates growth, survival and gene expression in human NK LDGL. ${ }^{19,20}$ Constitutive activation of ERK1/2 molecule was found in our patient's ANKL cells, confirming previous statements, although it remained unclear which upstream mediators led to ERK1/2 activation. Thus, the use of signalling pathway inhibitors might prove to be useful in elucidation of upstream signalling pathway in ANKL.

Patient's previous medical history revealed UC azathioprine therapy during the period of 17 months. It has been reported that patients who receive such treatment are at an increased risk of developing colorectal cancer. ${ }^{21}$ However, association of increased risk of lymphoma with immunosuppressive therapy of UC has not been unambiguously demonstrated. ${ }^{22,23}$ The meta-analysis performed by Kandiel et al. ${ }^{24}$ showed an approximately fourfold increased risk of lymphoma in UC patients treated with azathioprine. Caution is neces- sary with interpretation of these results, since an increased risk could be a consequence of the severity of the disease itself, or combination of both.

\section{Conclusions}

In conclusion, aggressive NK-cell leukaemia is chemotherapy resistant disease with extremely poor prognosis. However, leukaemic NK-cells resemble normal NK-cells in terms of P-gp functional capacity and expression of phosphorylated ERK1/2 signalling molecule. In this regard, drugs that block P-glycoprotein activity and activated signalling pathways might represent new means for targeted therapy.

\section{References}

1. Chan JKC, Quintanilla-Martinez L, Ferry JA, Peh SC. Extranodal NK/T-cell lymphoma, nasal type. In: Swerdlow $\mathrm{SH}$, Campo E, Harris NL et al, editors. WHO classification of tumours of haematopoietic and lymphoid tissues. 4th ed. Lyon, France: International agency for research on cancer (IARC); 2008. pp. 285-288.

2. Chan JKC, Jaffe ES, Ralfkiaer E, Ko YH.
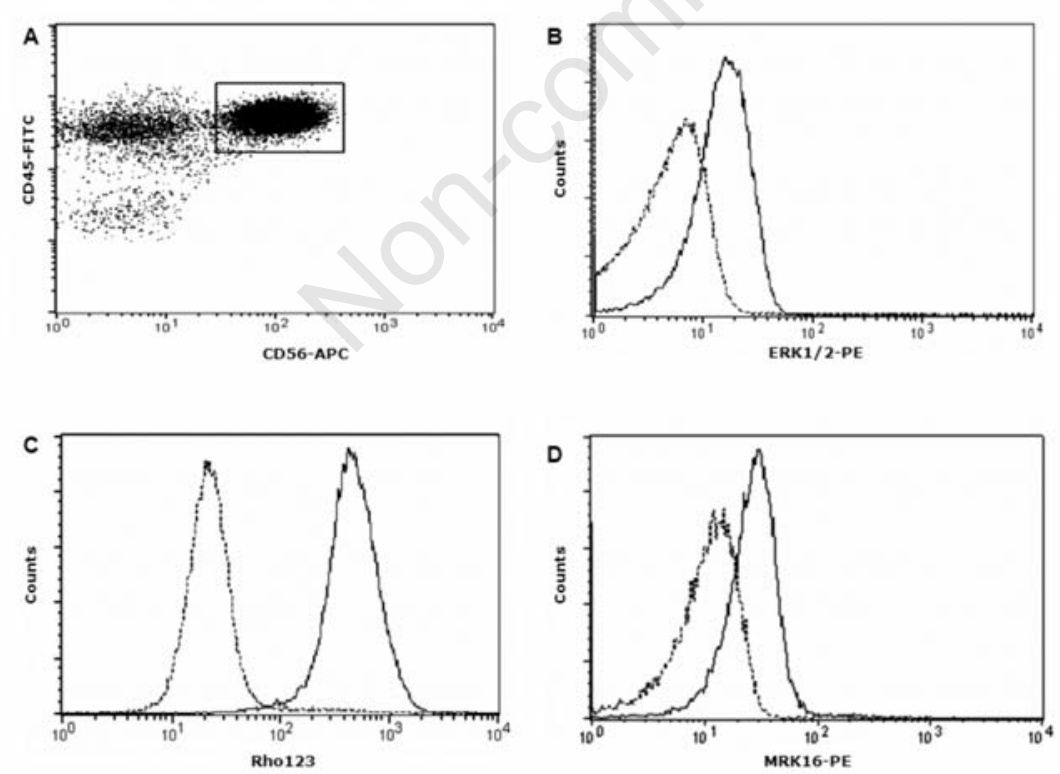

Figure 2. Analysis of NK-leukaemic cells. A) Gating of bone marrow CD56+ leukaemic cells for phenotypic and functional analysis; B) Antibody staining of phosphorylated ERK1/2 (solid line) in relation to isotypic control (dashed line); C) P-gp activity assessed by Rhodamine 123 efflux test: Rho123 efflux (dashed line) in relation to Rho123+ inhibitor (verapamil) (solid line); and D) P-gp expression by Mrk16 antibody staining (solid line) in relation to isotypic control (dashed line). Explanation in the text.

Aggressive NK-cell leukaemia. In: Swerdlow SH, Campo E, Harris NL et al, editors. WHO classification of tumours of haematopoietic and lymphoid tissues, 4th ed. Lyon, France: International agency for research on cancer (IARC); 2008. pp. 276277.

3. Armitage J, Vose J, Weisenburger D. International peripheral T-cell and natural killer/T-cell lymphoma study: pathology findings and clinical outcomes. J Clin Oncol 2008;26:4124-30.

4. Fernandez LA, Pope B, Lee C, Zayed E. Aggressive natural killer cell leukemia in an adult with establishment of NK cell line. Blood 1986;67:925-30.

5. Koizumi S, Seki H, Tachinami T, et al. Malignant clonal expansion of large granular lymphocytes with Leu-11+, Leu-7surface phenotype: in vitro responsiveness of malignant cells to recombinant human interleukin 2. Blood 1986;68:1065-73.

6. Hasserjian RP, Harris NL. NK-cell lymphomas and leukemias: a spectrum of tumors with variable manifestations and immunophenotype. Am J Clin Pathol 2007;127:860-8.

7. Sun HS, Su IJ, Lin YC, et al. A $2.6 \mathrm{Mb}$ interval on chromosome $6 \mathrm{q} 25.2-\mathrm{q} 25.3$ is commonly deleted in human nasal natural killer/T-cell lymphoma. $\mathrm{Br} \mathrm{J}$ Haematol 2003;122:590-9.

8. Suzuki R, Suzumiya J, Yamaguchi M, et al. Prognostic factors for mature natural killer (NK) cell neoplasms: aggressive NK cell leukemia and extranodal NK cell lymphoma, nasal type. Ann Oncol 2010;21: 1032-40.

9. Chaudhary PM, Mechetner EB, Roninson IB. Expression and activity of the multidrug resistance P-glycoprotein in human peripheral blood lymphocytes. Blood 1992;80:2735-9.

10. Egashira M, Kawamata N, Sugimoto K, et al. P-glycoprotein expression on normal and abnormally expanded natural killer cells and inhibition of P-glycoprotein function by Cyclosporin $\mathrm{A}$ and its analogue, PSC833. Blood 1999;93:599-606.

11. Drénou B, Lamy T, Amiot L, et al. CD3CD56+ non-Hodgkin's lymphomas with an aggressive behavior related to multidrug resistance. Blood 1997;89:2966-74.

12. Siu LL, Chan JK, Kwong YL. Natural killer cell malignancies: clinicopathological and molecular features. Histol Histopathol 2002;17:539-54.

13. Suzuki R, Suzumiya J, Nakamura S, et al. Aggressive natural killer-cell leukemia revisited: Large granular lymphocyte leukemia of cytotoxic NK cells. Leukemia 2004;18:763-70.

14. Ko YH, Park S, Kim K, et al. Aggressive natural killer cell leukemia: Is Epstein- 
Barr virus negativity an indicator of a favorable prognosis? Acta Haematol 2008;120:199-206.

15. Kwong YL. Natural killer-cell malignancies: diagnosis and treatment. Leukemia 2005;19:2186-94.

16. Wei S, Gilvary DL, Corliss BC, et al. Direct tumor lysis by NK cells uses a Ras-independent mitogen-activated protein kinase signal pathway. J Immunol 2000;165:38119.

17. Wei S, Gamero AM, Liu JH, et al. Control of lytic function by mitogen-activated protein kinase/extracellular regulatory kinase 2 (ERK2) in a human natural killer cell line: identification of perforin and granzyme B mobilization by functional ERK2. J Exp
Med 1998;187:1753-65.

18. Liang S, Zhang J, Wei H, et al. Differential roles of constitutively activated ERK1/2 and NF-kappa B in cytotoxicity and proliferation by human NK cell lines. Int Immunopharmacol 2005;5:839-48.

19. Djeu JY, Jiang K, Wei S. A view to a kill: signals triggering cytotoxicity. Clin Cancer Res 2002;8:636-40.

20. Epling-Burnette PK, Bai F, Wei S, et al. ERK couples chronic survival of NK cells to constitutively activated Ras in lymphoproliferative disease of granular lymphocytes (LDGL). Oncogene 2004;923:9220-9.

21. Ekbom A, Helmick C, Zack M, Adami HO. Ulcerative colitis and colorectal cancer - a population-based study. N Engl J Med.
1990; 323:1228-33.

22. Fraser AG, Orchard TR, Robinson EM, Jewell DP. Long-term risk of malignancy after treatment of inflammatory bowel disease with azathioprine. Aliment Pharmacol Ther 2002;16:1225-32.

23. Lewis JD, Bilker WB, Brensinger C, et al. Inflammatory bowel disease is not associated with an increased risk of lymphoma. Gastroenterology 2001;5:1080-7.

24. Kandiel A, Fraser AG, Korelitz BI, et al. Increased risk of lymphoma among inflammatory bowel disease patients treated with azathioprine and 6-mercaptopurine. Gut 2005;54:1121-5. 